\title{
Rebound Weight Gain Worsen the Experimental Non- Alcoholic Fatty Liver Disease in Rats
}

\author{
Noha M. Abogresha', Mona A Hussain ${ }^{2 *}$, Ranya Hassan³, Dalia A. El-Tamany ${ }^{4}$ \\ Departments of ${ }^{1}$ Physiology, Faculty of Medicine, Suez Canal University, Egypt, ${ }^{2}$ Physiology, Faculty of Medicine, \\ Port Said University, Egypt, ${ }^{3}$ Clinical pathology, Faculty of Medicine, Suez Canal University, Egypt. ${ }^{4}$ Home Econom- \\ ics, Nutrition and Food Science, Faculty of Education, Suez Canal University, Egypt
}

\begin{abstract}
Background: The prevalence of obesity is increasing worldwide and recurrent attempts for losing weight are very common. Diet cycling predisposes to health hazards including non-alcoholic fatty liver disease (NAFLD). Aim: to evaluate the effect of diet cycling on the course of NAFLD. Materials and methods: Seventy-two male albino Sprague-Dawly rats were used in the study. Rats were equally divided into three groups: Normal rat chow diet (NCD) control group, high fat diet (HFD) group, and diet cycling group. NCD and HFD groups were kept on NCD and HFD respectively for the overall experimental period. Diet cycling group was fed with HFD for eight weeks to induce NAFLD, and then shifted to NCD for four weeks, followed by HFD for four weeks to allow rebound weight gain. By the end of eight, twelve and sixteen weeks, eight rats from each group were randomly selected and evaluated for body mass index (BMI), liver index, lipid profile, free fatty acids, liver enzymes, homeostasis model assessment index for insulin resistance (HOMA-IR) index, tumor necrosis factor alpha (TNF- $\alpha$ ), interleukin- 6 (IL-6), and transforming growth factor- $\beta 1$ (TGF$\left.\beta_{1}\right)$.+ Hepatic oxidative stress enzymes were also measured. Liver histopathology and alphasmooth-muscle actin (a-SMA) immunoreactivity were evaluated. Results: Diet cycling group showed significant increase in BMI, Liver index, serum inflammatory markers, and concomitant significant decrease in hepatic glutathione peroxidase levels. In addition, marked fatty and inflammatory infiltrations were observed in diet cycling group. Conclusion: Diet cycling had a negative influence on NAFLD and interfered with normal liver function. These results suggest that rebound weight gain negatively affect the course of NAFLD.
\end{abstract}

Keywords: NAFLD, HFD, rebound weight gain, diet cycling

\section{Introduction}

Nonalcoholic fatty liver disease (NAFLD) is one of the most common forms of liver diseases worldwide ${ }^{(1-4)}$. The prevalence of NAFLD may be as high as $76 \%$ in obese subjects ${ }^{(5)}$. The biological mechanism underlying steatosis occurrence and progression to non-alcoholic steatohepatitis is not entirely understood. A two-hit hypothesis has been proposed ${ }^{(6)}$; in which a first hit is able to induce liver fat accumulation and a second hit prompts steatosis progression to non-alcoholic steatohepatitis. The most widely supported theory implicates insulin resistance as the key mechanism in primary NAFLD, leading to hepatic steatosis. The presumed factors initiating second hit are oxidative stress and subsequent lipid peroxidation, proinflammatory cytokines (principally TNF- $\alpha$ ) and hormones derived from adipose tissue (adipocytokines) ${ }^{(7)}$. It is 
reported that chronic increase in dietary fat intake greatly affects the liver ${ }^{(8-10)}$. Unfortunately, high fat diet (HFD) can cause NAFLD through either eliciting hepatic steatosis; reducing hepatic insulin sensitivity or increasing hepatic oxidative stress, leading to lipid peroxidation and inflammation in the hepatocytes ${ }^{(10)}$, proinflammatory cytokines may also have a role ${ }^{(6)}$. NAFLD encompasses a spectrum of liver pathology from isolated steatosis to nonalcoholic steatohepatitis, where fat accumulation is associated with inflammation and/or evidence of cellular injury and cirrhosis $^{(11)}$. Caloric restriction is considered the most effective tool for reducing the body fat mass but, recently, much has been said about obesity and the difficulties in maintaining long-term body weight control. In addition, the increasing lean model has induced many people to make frequent attempts at dieting ${ }^{(12)}$. Therefore, studies on the effect of weight cycling on obesity induced NAFLD are very important. So, the aim of this study was to address the effect of diet cycling and rebound weight gain on the pathogenesis of NAFLD in rats.

\section{Materials and Methods}

\section{Experimental animal}

Seventy-two male albino Sprague-Dawly rats, obtained from the Egyptian Organization for Biological Products and Vaccines (Cairo, Egypt), were used in the current study. Rats had an initial body weight in the range of 120-150 g. All rats were left to acclimatize for one week prior to the experiment and were housed in plastic cages maintained at controlled room temperature (22-24 C) with 12 hour diurnal (day and night change) with free access to normal chow diet which contains $67 \%$ carbohydrates, $10 \%$ fat, and $23 \%$ protein as the energy sources (overall calorie: $3.6 \mathrm{kcal} / \mathrm{g}$ ) and tap water. All experimental protocols were approved by the Animal Care and Use Committee at the Faculty of Medicine, Suez Canal University.

\section{Experimental design}

Rats were randomly divided into three equal groups; $(n=24$ rats). Normal rat chow $\operatorname{diet}(N C D)$ group, which served as a control group and was maintained on NCD throughout the experimental period of 16 weeks. High fat diet (HFD) group, which was maintained on a HFD for 16 weeks. Diet cycling group which was fed HFD for 8 weeks, followed by NCD for 4 weeks and followed by HFD for 4 weeks. The HFD was prepared by mixing $2 \mathrm{~g}$ cholesterol, $10 \mathrm{~g}$ lard oil and $88 \mathrm{~g}$ normal pellet chow. The HFD was composed of the following energy sources: $52 \%$ was provided by carbohydrates, $30 \%$ by fat, and $18 \%$ by protein (overall calories: $4.8 \mathrm{kcal} / \mathrm{g}$ ). The NCD was composed of the following energy sources: $67 \%$ carbohydrates, $10 \%$ fat, and $23 \%$ protein (overall calorie: $3.6 \mathrm{kcal} / \mathrm{g})^{(13)}$.

\section{Blood and tissue sampling}

By the end of eight, twelve, and sixteen weeks, eight rats from each group were fasted overnight. The body weight and length were measured. BMI was calculated according the formula (BMI = body weight (g)/length $\left.{ }^{2}\left(\mathrm{~cm}^{2}\right)\right)^{(14)}$. Rats were anesthetized by ketamine ( $80 \mathrm{mg} / \mathrm{kg}$, i.p.) and blood samples were collected by cardiac puncture. The collected samples were centrifuged for $3 \mathrm{~min}$ at $1000 \times \mathrm{g}$ to obtain the serum, which was then stored at $-80{ }^{\circ} \mathrm{C}$. The liver was rapidly dissected, washed with ice cold $0.9 \% \mathrm{NaCl}$ solution, weighed and the liver index was calculated (liver weight/body weight $\times 100$ ). One part of the liver (0.3g) was then blotted and finally kept at $-80^{\circ} \mathrm{C}$. Liver samples were taken 5 $\mathrm{mm}$ away from the edge of the largest hepatic lobe, fixed with $10 \%(\mathrm{v} / \mathrm{v})$ formaldehyde, embedded in paraffin wax, and 
stained with hematoxylin and eosin (H\&E) and Masson's trichrome stain.

Measurement of serum biochemical parameters

Serum activity of alanine transaminase (ALT) and aspartic transaminase (AST) were spectrophotometrically measured using commercial kits (Biocon Diagnostic, Germany). Glucose, Total cholesterol (TC), and triglycerides (TG) measurements were performed with commercial kits on the Cobas c311 autoanalyzer (Roche Diagnostics, Germany). Free fatty acids assay was performed with BioVision's Free Fatty Acid Quantification Colorimetric Kit. ELISA kits for serum levels of TNF- $\alpha$, IL-6, IL-10 (Ray Biotech Inc., Norcross, USA), TGF- $\beta 1$ (Boster Biological technology, Wuhan, China) and insulin (Crystal Chem Inc., Downers Grove, IL 60515, USA) were used. The assays were carried out according the instructions of the manufacturer using an automated ELISA reader (Europe S.A. Belgium). Insulin resistance was determined using the homeostasis model assessment index for insulin resistance (HOMA-IR) using the following formula: HOMA-IR index= [fasting glucose $(\mathrm{mmol} / \mathrm{L}) \times$ fasting insulin $(\mu \mathrm{U} / \mathrm{ml})] / 22.5)^{(15)}$.

Measurement of hepatic oxidative stress enzymes:

Oxidative stress markers: Lipid peroxidase, superoxide dismutase, catalase (CAT) and glutathione peroxidase were measured in liver homogenate. Tissue samples were homogenized in four volumes of ice-cold Tris- $\mathrm{HCl}$ buffer ( $50 \mathrm{mM}, \mathrm{pH} 7.4$ ) on homogenizer (Ultra Turrax IKA T18 Basic, USA) for $2 \mathrm{~min}$ at $5,000 \mathrm{~g}$ at $4^{\circ} \mathrm{C}$. Then the level of lipid peroxidase was measured using the method of Draper ${ }^{(16)}$. Superoxide dismutase (SOD) level was measured according to the method of Woolliams ${ }^{(17)}$. CAT activity was quantified in liver tissue by the method of Aebi ${ }^{(18)}$. Glutathione peroxidase level was measured according to the method described by Beutler ${ }^{(19)}$. All assays were spectrophotometrically done using UV-visible spectrophotometer (UV-1601PC, Shimadzu, Japan).

\section{Histopathological examination}

All histological examinations were performed by an experienced pathologist who was blinded to the experiment groups. The paraffin-embedded liver tissues were cut into 4- $\mu \mathrm{m}$ sections, and standard $\mathrm{H} \& \mathrm{E}$ staining was performed. Hepatic fibrosis was assessed by Masson stain. Ten 200x light microscopic fields were viewed on each section and scored for the severity of hepatic steatosis, inflammation and fibrosis according to the following criteria: For hepatic steatosis: grade 0 , no fat; grade 1 , steatosis occupying less than 33\% of the hepatic parenchyma; grade 2 , steatosis occupying $34-66 \%$ of the hepatic parenchyma and grade 3 , steatosis occupying more than $66 \%$ of the hepatic parenchyma. For inflammatory cell infiltration: grade 0 : none; grade 1, 1-2 foci/field; grade 2, 3-4 foci/field; grade 3, more than 4 foci/field. The staging of hepatic fibrosis was investigated by Masson's trichrome staining as follows: 0 , none; 1 , mild, zone 3 , perisinusoidal; 2 , moderate, zone 3, perisinusoidal; 3 , portal/periportal; 4, bridging fibrosis ${ }^{(20)}$.

\section{Immunostaining}

Paraffin-embedded liver tissues were deparaffinized with xylene series, washed well with graded ethanol and permeabilized with $0.2 \%$ trypsin at room temperature for $30 \mathrm{~min}$. After inactivation of endogenous peroxidase by $3 \% \mathrm{H}_{2} \mathrm{O}_{2}$ for 20 min, tissue sections were washed with phosphate-buffered saline (PBS) and reacted with mouse anti-rat alpha-smoothmuscle actin (a-SMA) (Sigma, Ltd., St Louis, $\mathrm{MO}$, at dilution 1:200. PBS replaced the primary antibody as a negative control. Following primary antibody incubation, the 
tissue sections were washed in PBS and incubated with secondary antibody for 30 min at room temperature. The tissue sections were then washed with PBS, reacted with $0.01 \% \mathrm{H}_{2} \mathrm{O}_{2}$ and 3, 3' diaminobenzidine (DAB; Dako, Denmark) at room temperature for 3-5 min, washed again with PBS and covered with glycerin gelatin for light microscopy ${ }^{(13)}$.

\section{Statistical Analysis}

Data were expressed as mean \pm SEM unless otherwise specified, and analyzed using the Statistical Package of Social Sciences (SPSS program, version 17, SPSS Inc., Chicago, IL, USA). The difference of mean values among groups was assessed by one-way analysis of variance (ANOVA) followed by Bonferroni's multiple comparisons test. $P$ value $<0.05$ was considered significant.
Effects of HFD on body weight, liver weight, $B M I$ and liver index

HFD group at 8, 12 and 16 weeks had significant increase, in body weight, liver weight, BMI and liver index versus NCD control group ( $p<0.05)$. At 8 weeks, the diet cycling group showed a significant increase in body weight, liver weight, BMI and liver index versus NCD control group at 8,12 and 16 weeks. At 12 weeks, the diet cycling group had a significant decrease in body weight, BMI and liver index versus HFD group at 8,12 and 16 weeks. At 16 weeks, the diet cycling group showed a significant increase in body weight and BMI versus NCD control, HFD groups at 8, 12 and 16 weeks and diet cycling group at 8 weeks. Also, diet cycling group at 16 weeks had a significant increase in the liver weight and index versus NCD control group at 8, 12 and 16 weeks $(p<0.05)$. (Table 1$)$.

\section{Results}

Table 1: Weight, Length, BMI, Liver Weight, Liver Weight Index

\begin{tabular}{|l|cccccc|}
\hline \multirow{3}{*}{ Group } & Weeks \# & Weight $(\mathrm{g})$ & Length $(\mathrm{cm})$ & Liver weight $(\mathrm{g})$ & BMI & $\begin{array}{c}\text { Liver wt } \\
\text { index }\end{array}$ \\
\hline Control & 8 weeks & $145 \pm 17.3$ & $19.0 \pm 1.15$ & $4.45 \pm 0.87$ & $0.40 \pm 0.007$ & $2.745 \pm 0.15$ \\
& 12 weeks & $149.3 \pm 0.5$ & $19.67 \pm 0.58$ & $4.83 \pm 0.23$ & $0.38 \pm 0.02$ & $2.24 \pm 0.14$ \\
& 16 weeks & $158.3 \pm 36.8$ & $19.00 \pm 1.0$ & $5.03 \pm 1.76$ & $0.43 \pm 0.06$ & $2.79 \pm 0.41$ \\
\hline HFD & 8 weeks & $238.5 \pm 26.1^{*}$ & $18.25 \pm 1.5$ & $7.58 \pm 0.80^{*}$ & $0.93 \pm 0.05^{*}$ & $5.67 \pm 0.57^{*}$ \\
& 12 weeks & $254.6 \pm 10.3^{*}$ & $21.30 \pm 0.45$ & $7.90 \pm 1.87^{*}$ & $1.15 \pm 0.03^{*}$ & $6.71 \pm 1.06^{*}$ \\
& 16 weeks & $300.6 \pm 34.2^{*}$ & $21.3 \pm 2.08$ & $9.5 \pm 1.70^{*}$ & $1.33 \pm 0.05^{*}$ & $8.68 \pm 0.21^{*}$ \\
\hline Cycling & 8 weeks & $230.25 \pm 27.9^{*}$ & $18.75 \pm 0.50$ & $7.475 \pm 0.71^{*}$ & $1.19 \pm 0.05^{*}$ & $5.59 \pm .059^{*}$ \\
& 12 weeks & $176.33 \pm 6.51 \dagger$ & $20.0 \pm 1.0$ & $5.0 \pm 0.26$ & $0.54 \pm 0.04 \dagger$ & $3.4 \pm 0.21^{\dagger}$ \\
& 16 weeks & $369.8 \pm 9.7^{*} \dagger$ & $20.0 \pm 1.09$ & $8.97 \pm 1.52^{*}$ & $1.92 \pm 0.01^{*}+\$$ & $7.79 \pm 0.37^{*}$ \\
\hline
\end{tabular}

All data are expressed as mean \pm SEM. and were analyzed using one-way ANOVA and Bonferronii's post-hoc test. *=Cycling and HFD group compared to control group 8,12,16 weeks at $P<0.05 . \dagger=C y c l i n g$ group compared to HFD 8,12,16 weeks group at $\mathrm{P}<0.05$. . $\$=$ Cycling group 16 weeks compared to cycling group 8 weeks at $\mathrm{P}<0.05$. @= liver weight/body weight $\times 100$

\section{Serum biochemical parameters}

i) Liver functions: At 8 weeks, on comparison to control group, serum AST was significantly higher in HFD and diet cycling groups $(p<0.05)$. At 12 weeks, HFD group showed significant increase in serum AST and ALT concentrations versus NCD and diet cycling groups $(p<0.05)$. At 16 weeks, diet-cycling group showed significant increase in serum AST and ALT when compared to both NCD and HFD groups. In addition, HFD group had a significant increase in serum AST and ALT concentrations versus NCD group ( $p<0.05$, Table 2$)$. 
Table 2: Liver Functions, Serum Fasting Insulin, Fasting Blood

\begin{tabular}{|l|cccccc|}
\hline Group & Weeks \# & $\begin{array}{c}\text { AST } \\
(\mathrm{mg} / \mathrm{dL})\end{array}$ & $\begin{array}{c}\text { ALT } \\
(\mathrm{mg} / \mathrm{dL})\end{array}$ & $\begin{array}{c}\text { HOMA-IR } \\
(\mathrm{U} / \mathrm{dL})\end{array}$ & $\begin{array}{c}\text { Fasting insulin } \\
(\mathrm{pmol} / \mathrm{L})\end{array}$ & $\begin{array}{c}\text { Fasting blood } \\
\text { glucose } \\
(\mathrm{mg} / \mathrm{dL})\end{array}$ \\
\hline Control & 8 weeks & $152 \pm 61.1$ & $40.5 \pm 28.2$ & $0.9 \pm 4.5$ & $7.6 \pm 1.53$ & $78.5 \pm 17.8$ \\
& 12 weeks & $145.6 \pm 76.9$ & $48.67 \pm 7.2$ & $1.2 \pm 0.9$ & $7.1 \pm 3.6$ & $73.6 \pm 37.8$ \\
& 16 weeks & $105.1 \pm 97.0$ & $50.67 \pm 19.7$ & $0.87 \pm 0.17$ & $3.3 \pm 1.3$ & $73.3 \pm 56.8$ \\
\hline HFD & 8 weeks & $276.0 \pm 37.1^{*}$ & $60.0 \pm 5.4$ & $6.0 \pm 1.0^{*}$ & $27.0 \pm 3.7^{*}$ & $115.7 \pm 18.5^{*}$ \\
& 12 weeks & $243.2 \pm 50.2^{*}$ & $85.0 \pm 17.7^{*}$ & $7.6 \pm 0.8^{*}$ & $26.4 \pm 3.1^{*}$ & $102.2 \pm 10.7^{*}$ \\
& 16 weeks & $212.6 \pm 35.9^{*}$ & $82.6 \pm 11.01^{*}$ & $6.5 \pm 0.1^{*}$ & $25.5 \pm 0.5^{*}$ & $93.3 \pm 16.4^{*}$ \\
\hline Cycling & 8 weeks & $293.0 \pm 43.6^{*}$ & $63.0 \pm 5.8$ & $5.5 \pm 1.2^{*}$ & $29.0 \pm 4.0^{*}$ & $110.0 \pm 12.3^{*}$ \\
& 12 weeks & $192.3 \pm 23.7^{* \dagger}$ & $42.0 \pm 2.6^{\dagger}$ & $4.9 \pm 2.2^{*}$ & $10.8 \pm 36.4^{*}$ & $70.3 \pm 59.4 \dagger$ \\
& 16 weeks & $283.9 \pm 47.1^{* \dagger}$ & $160 \pm 32.0^{*+\$}$ & $10.0 \pm 0.7^{*+\$}$ & $37.4 \pm 3.6^{*}$ & $93.3 \pm 34.9^{*}$ \\
\hline
\end{tabular}

All data are expressed as mean \pm SEM and were analyzed using one-way ANOVA and Bonferronii's post-hoc test. * =Cycling and HFD group vs. control group 8,12,16 weeks at $p<0.05 . \dagger=$ Cycling group vs. HFD 8,12,16 weeks group at $p<0.05$. $\$=$ Cycling group 16 weeks vs. cycling group 8 weeks at $p<0.05$.

ii) Fasting blood glucose, Fasting Insulin concentrations and HOMA-IR index

Compared to control group, fasting blood glucose, fasting serum insulin concentrations and HOMA-IR showed significant increase in HFD group at 8 weeks, 12 and 16 weeks. Diet cycling group showed significant increase in fasting serum insulin concentration and HOMA-IR index at 8, 12 and 16 weeks and a significant increase in fast

ing serum glucose concentration at 8 and 16 weeks versus NCD group. Fasting serum glucose concentration was significantly lower in diet cycling group at 12 weeks versus HFD group. Fasting serum insulin concentration and HOMA-IR index were significantly higher in diet cycling group at 16 weeks than HFD group and diet cycling group at 8 weeks. (Table 2 ).

Table 3: Serum Triglycerides, T. cholesterol, TNF- $\alpha$, IL-6, IL-10 and TGF- $\beta$ levels

\begin{tabular}{|c|c|c|c|c|c|c|}
\hline $\begin{array}{l}\text { Groups } \\
\text { Weeks \# }\end{array}$ & $\begin{array}{l}\text { Triglycer- } \\
\text { ides }(\mathrm{mg} / \mathrm{dl})\end{array}$ & $\begin{array}{l}\text { T. choles- } \\
\text { terol } \\
\text { (mg/dl) }\end{array}$ & $\begin{array}{l}\text { TNF- } \alpha \\
(\mathrm{pg} / \mathrm{ml})\end{array}$ & $\begin{array}{c}\text { IL-6 } \\
(\mathrm{pg} / \mathrm{ml})\end{array}$ & $\begin{array}{c}\text { IL-10 } \\
(\mathrm{pg} / \mathrm{ml})\end{array}$ & $\begin{array}{l}\text { TGF- } \beta \\
(\mathrm{ng} / \mathrm{ml})\end{array}$ \\
\hline \multicolumn{7}{|l|}{ Control } \\
\hline 8 weeks & $69.5 \pm 1.7$ & $49.0 \pm 2.3$ & $28.60 \pm 2.65$ & $36.65 \pm 4.45$ & $172.3 \pm 4.8$ & $0.013 \pm 0.003$ \\
\hline 12 weeks & $62.0 \pm 2.0$ & $54.3 \pm 7.2$ & $39.8 \pm 10.1$ & $42.2 \pm 9.79$ & $168.9 \pm 9.4$ & $0.014 \pm 0.004$ \\
\hline 16 weeks & $58.0 \pm 27.0$ & $51.0 \pm 20.4$ & $33.4 \pm 20.9$ & $40.56 \pm 11.0$ & $132.1 \pm 19.5$ & $0.011 \pm 0.004$ \\
\hline \multicolumn{7}{|l|}{ HFD } \\
\hline$\overline{8 \text { weeks }}$ & $184.0 \pm 37.0^{*}$ & $98.7 \pm 15.2^{*}$ & $88.0 \pm 25.9^{*}$ & $98.18 \pm 3.97 *$ & $65.9 \pm 11.2^{*}$ & $0.047 \pm 0.001^{*}$ \\
\hline 12 weeks & $241.2 \pm 86.5^{*}$ & $119.8 \pm 30.4^{*}$ & $105.0 \pm 14.3^{*}$ & $96.2 \pm 31.7^{*}$ & $59.2 \pm 24.3^{*}$ & $0.047 \pm 0.002^{*}$ \\
\hline 16 weeks & $248.6 \pm 33.1^{*}$ & $172.6 \pm 4.9 *$ & $113.6 \pm 4.1^{*}$ & $112.4 \pm 17.0 *$ & $72.9 \pm 10.7^{*}$ & $0.042 \pm 0.001^{*}$ \\
\hline \multicolumn{7}{|l|}{ Cycling } \\
\hline 8 weeks & $189.0 \pm 37.0^{*}$ & $92.6 \pm 4.9^{*}$ & $72.4 \pm 18.5^{*}$ & $111.2 \pm 15.8 *$ & $79.9 \pm 14.0^{*}$ & $0.046 \pm 0.002^{*}$ \\
\hline 12 weeks & $55.3 \pm 16.7^{\dagger}$ & $78.3 \pm 5.5^{\dagger}$ & $97.9 \pm 13.2^{*}$ & $103.2 \pm 32.6 *$ & $87.2 \pm 14.6^{* \dagger}$ & $0.047 \pm 0.004^{*}$ \\
\hline 16 weeks & $227.3 \pm 41.6^{*}$ & $153.8 \pm 37.9^{*}$ & $146.5 \pm 13.5^{*+\$}$ & $119.0 \pm 10.1 *$ & $74.4 \pm 15.7^{*}$ & $0.045 \pm 0.002^{*}$ \\
\hline
\end{tabular}

All data are expressed as mean \pm SEM and were analyzed using one-way ANOVA and Bonferronii's post-hoc test. * =Cycling and HFD group vs. control group 8,12,16 weeks at $p<0.05 . \dagger=$ Cycling group vs. HFD 8,12,16 weeks group at $p<0.05 . . \$=$ Cycling group 16 weeks vs. cycling group 8 weeks at $p<0.05$.

iii) Serum lipids and inflammatory markers 1- Serum TG and TC concentrations
At 8 weeks, compared to control group, serum TG and TC were significantly higher 
in HFD group and diet cycling group ( $p<$ 0.05). At 12 weeks, HFD group showed significant increase in serum TG and TC concentrations versus NCD and diet cycling groups $(p<0.05)$. At 16 weeks, serum TC and TC levels showed significant increase in HFD and diet cycling groups versus NCD group $(p<0.05)$ (Table 3$)$.

\section{2- Serum TNF- $\alpha$, IL-6, IL-10 and TGF- 8 levels} Compared to NCD group, TNF- $\alpha$, IL- 6 and TGF- $\beta$ levels showed significant increase in HFD and diet cycling groups at 8,12 and 16 weeks. IL-10 levels showed a significant decrease in HFD and diet cycling groups versus NCD group at 8,12 and 16 weeks. At 12 weeks, IL-10 level was significantly higher in diet cycling group versus HFD group ( $p<0.05$, Table 3 ).

Oxidative stress markers in liver tissue and serum Free Fatty Acids (FFA)
Compared to control group, the level of lipid peroxidase was significantly higher and glutathione peroxidase level was significantly lower in the HFD and diet cycling groups at 8,12 and 16 weeks. At 16 weeks, the level of glutathione peroxidase was significantly decreased in diet cycling group versus HFD group and diet cycling group at 8 weeks. Regarding FFA levels; at 8 weeks, compared to control group, serum FFA level was significantly higher in HFD and diet cycling groups $(p<0.05)$. At 12 weeks, serum FFA level was significantly higher in HFD than NCD and diet cycling groups ( $p<0.05)$. At 16 weeks, serum FFA level was significantly higher in HFD and diet-cycling groups versus NCD group and the increase in serum FFA concentrations in diet cycling group was significantly higher than HFD group and diet cycling group at 8 weeks. (Table 4).

Table 4: Hepatic Oxidative Stress Enzymes, FFA Levels

\begin{tabular}{|c|c|c|c|c|c|}
\hline $\begin{array}{l}\text { Group } \\
\text { Weeks \# }\end{array}$ & $\begin{array}{l}\text { Superoxide } \\
\text { (u/gm. LT) }\end{array}$ & $\begin{array}{l}\text { Lipid Peroxide } \\
\text { (nmol/gm. LT) }\end{array}$ & $\begin{array}{l}\text { Catalase } \\
\text { (u/gm. LT) }\end{array}$ & $\begin{array}{l}\text { Glutathione } \\
\text { (u/gm. LT) }\end{array}$ & $\begin{array}{l}\text { FFA levels } \\
(\mathrm{mmol} / \mathrm{L})\end{array}$ \\
\hline \multicolumn{6}{|l|}{ Control } \\
\hline 8 weeks & $262.50 \pm 43.30$ & $31.84 \pm 1.20$ & $1.23 \pm .06$ & $156.99 \pm 24.04$ & $0.75 \pm 0.14$ \\
\hline 12 weeks & $250.33 \pm 43.02$ & $26.28 \pm 5.40$ & $1.28 \pm 0.59$ & $163.93 \pm 24.04$ & $0.76 \pm 0.13$ \\
\hline 16 weeks & $287.50 \pm 57.28$ & $26.28 \pm 5.41$ & $1.28 \pm 0.59$ & $197.26 \pm 33.69$ & $0.79 \pm 0.18$ \\
\hline \multicolumn{6}{|l|}{$\underline{\mathrm{HFD}}$} \\
\hline 8 weeks & $309.38 \pm 18.5$ & $46.44 \pm 3.69^{*}$ & $1.09 \pm 0.11$ & $106.72 \pm 67.39^{*}$ & $0.98 \pm 0.15^{*}$ \\
\hline 12 weeks & $315.0 \pm 33.54$ & $48.91 \pm 5.12^{*}$ & $1.24 \pm 0.11$ & $122.91 \pm 17.12^{*}$ & $1.02 \pm 0.17^{*}$ \\
\hline 16 weeks & $337.5 \pm 0.001$ & $79.89 \pm 3.32^{*}$ & $1.19 \pm 0.03$ & $85.39 \pm 11.23^{*}$ & $1.1 \pm 0.2^{*}$ \\
\hline \multicolumn{6}{|l|}{ Cycling } \\
\hline 8 weeks & $318.75 \pm 21.65$ & $56.44 \pm 3.69^{*}$ & $1.09 \pm 0.11$ & $104.72 \pm 67.38^{*}$ & $0.96 \pm 0.11$ \\
\hline 12 weeks & $300.0 \pm 37.5$ & $42.23 \pm 1.71^{*}$ & $1.23 \pm 0.12$ & $114.74 \pm 2.83^{*}$ & $0.88 \pm 0.14^{\dagger}$ \\
\hline 16 weeks & $300.0 \pm 0.01$ & $68.27 \pm 5.29^{*}$ & $1.18 \pm 0.10$ & $58.36 \pm 0.00^{*+\$}$ & $1.37 \pm 0.42^{*+\$}$ \\
\hline
\end{tabular}

Data are expressed as mean \pm SEM, and were analyzed using one-way ANOVA and Bonferronii's post-hoc test. ${ }^{*}=$ Cycling and HFD group vs. control group 8,12,16 weeks at $p<0.05 . \dagger=$ Cycling group vs. HFD 8,12,16 weeks group at $p<0.05$. $\$=$ Cycling group 16 weeks vs. cycling group 8 weeks at $p<0.05$. LT=liver tissue.

\section{Liver histopathological results}

Eight and twelve weeks of HFD feeding in rats in duced grade 1 (mild) steatosis with mild lobular and perisinusoidal inflammation. At the end of 16 weeks, HFD group showed grade 2 steatohepatitis, and diet cycling group revealed a more advanced grade of steatosis (grade 3 ) with grade 2 hepatitis (Table 5 and figure $1 A$ ). Both, diet cycling group at 16 weeks and HFD group at 12 and 16 weeks shared comparable stages of fibrosis (Table 5 and figure 1B). 
The livers of rats in HFD group at 12 and 16 weeks showed significant increase in $a$ SMA immunoreactivity with marked perisinusoidal distribution versus control group. These results were compatible with those of diet cycling group at16 weeks (Figure 2).

Table 5: Hepatic histopathological score

\begin{tabular}{|c|c|c|c|c|c|c|c|c|}
\hline \multirow[t]{2}{*}{ Groups } & \multirow{2}{*}{ Weeks \# } & \multirow{2}{*}{$\begin{array}{l}\text { Steatosis } \\
\text { score }\end{array}$} & \multirow{2}{*}{$\begin{array}{l}\text { Inflammation } \\
\text { score }\end{array}$} & \multicolumn{5}{|c|}{ Fibrosis stage } \\
\hline & & & & 0 & 1 & 2 & 3 & 4 \\
\hline \multirow[t]{3}{*}{ Control } & 8 weeks & 0 & 0 & 8 & $\overline{0}$ & 0 & 0 & $\overline{0}$ \\
\hline & 12 weeks & 0 & 0 & 8 & 0 & 0 & 0 & 0 \\
\hline & 16 weeks & 0 & 0 & 8 & 0 & 0 & 0 & 0 \\
\hline \multirow[t]{3}{*}{ HFD } & 8 weeks & $0.67 \pm 0.33^{*}$ & $0.33 \pm 0.12^{*}$ & 6 & 2 & 0 & 0 & 0 \\
\hline & 12 weeks & $1.6 \pm 0.35^{*}$ & $1.90 \pm 0.87^{*}$ & 6 & 1 & 1 & 0 & 0 \\
\hline & 16 weeks & $2.61 \pm 0.2^{*}$ & $2.5 \pm 0.70 *$ & 0 & 2 & 6 & 0 & 0 \\
\hline \multirow[t]{3}{*}{ Cycling } & 8 weeks & $0.70 \pm 0.25^{*}$ & $0.47 \pm 0.11^{*}$ & 6 & 2 & 0 & 0 & 0 \\
\hline & 12 weeks & $0.33 \pm 0.51^{\dagger}$ & $1.60 \pm 0.26^{*}$ & 6 & 2 & 0 & 0 & 0 \\
\hline & 16 weeks & $3.3 \pm 0.7^{*+\$}$ & $3.8 \pm 0.52^{*+\$}$ & 5 & 2 & 1 & 0 & 0 \\
\hline
\end{tabular}

Data are expressed as mean \pm SEM and were analyzed using one-way ANOVA and Bonferronii's post-hoc test. *=Cycling and HFD group vs. control group 8,12,16 weeks at $p<$ 0.05 . $\dagger=$ Cycling group vs. HFD 8,12,16 weeks group at $p<0.05$. $\$=$ Cycling group 16 weeks vs. cycling group 8 weeks at $p<0.05 . n=24$

\section{Discussion}

Although weight cycling is commonly occurred in overweight and obese population, its consequence on the liver is still debated. It is not clear if weight cycling poses greater risk than obesity maintenance or not. The present work assessed the effect of diet cycling on the established NAFLD. Several studies reported that NAFLD severity is proportional to HFD intake ${ }^{(13,21-24)}$. So, in order to simulate the pathophysiological alterations that happened in real NAFLD, we selected the HFD model as was demonstrated previously ${ }^{(13)}$. In the present study, eight weeks of HFD feeding in rats induced grade 1 (mild) steatosis with mild lobular and perisinusoidal inflammation. Other studies, reported diffuse mixed hepatic steatosis after HFD for four weeks ${ }^{(13)}$, six weeks $^{(26)}$, or twelve weeks ${ }^{(27)}$. Our results revealed that HFD feeding for 8 weeks induced a marked elevation in the BMI, liver index, liver weight and activities of liver en zymes, several studies reported similar results $^{(26-30)}$. In the current study, shifting to NCD in diet cycling group for 4 weeks almost reversed these changes dramatically. Unfortunately, returning back to HFD in the diet cycling group markedly increased BMI, liver weight and index and liver enzymes, and in this time, these changes were stronger in diet cycling at 16 weeks than HFD group at 16 weeks and diet cycling group at 8 weeks. The aggressive weight regain in the diet-cycling group in our study is supported by both rat diet cycling models and human weight regains data. This finding may be due to persisted energy gap and increased drive to eat after diet shifting ${ }^{(31-32)}$. Also weight regain may induce more rapid adipose tissue growth and hyperplasia due to metabolic shifts favoring lipid storage ${ }^{(33)}$. The marked increase in liver weight, index, and enzymes with diet cycling reflected severe liver injury. 
A)
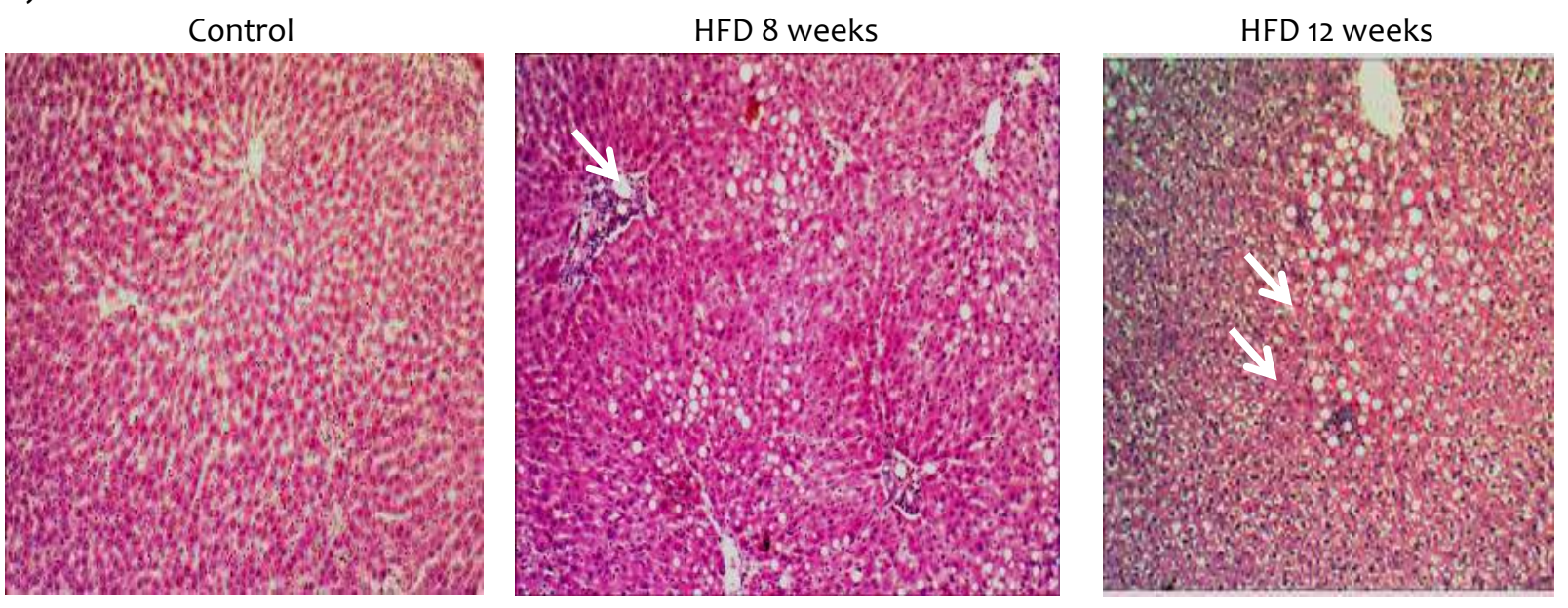

HFD 16 weeks

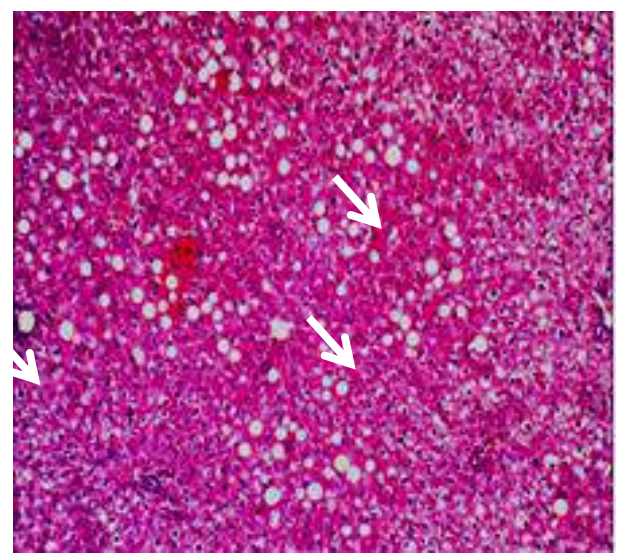

Cycling 16 weeks

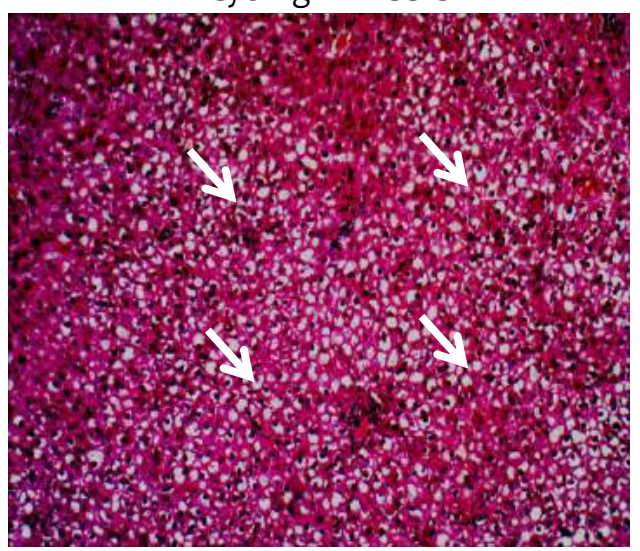

B)
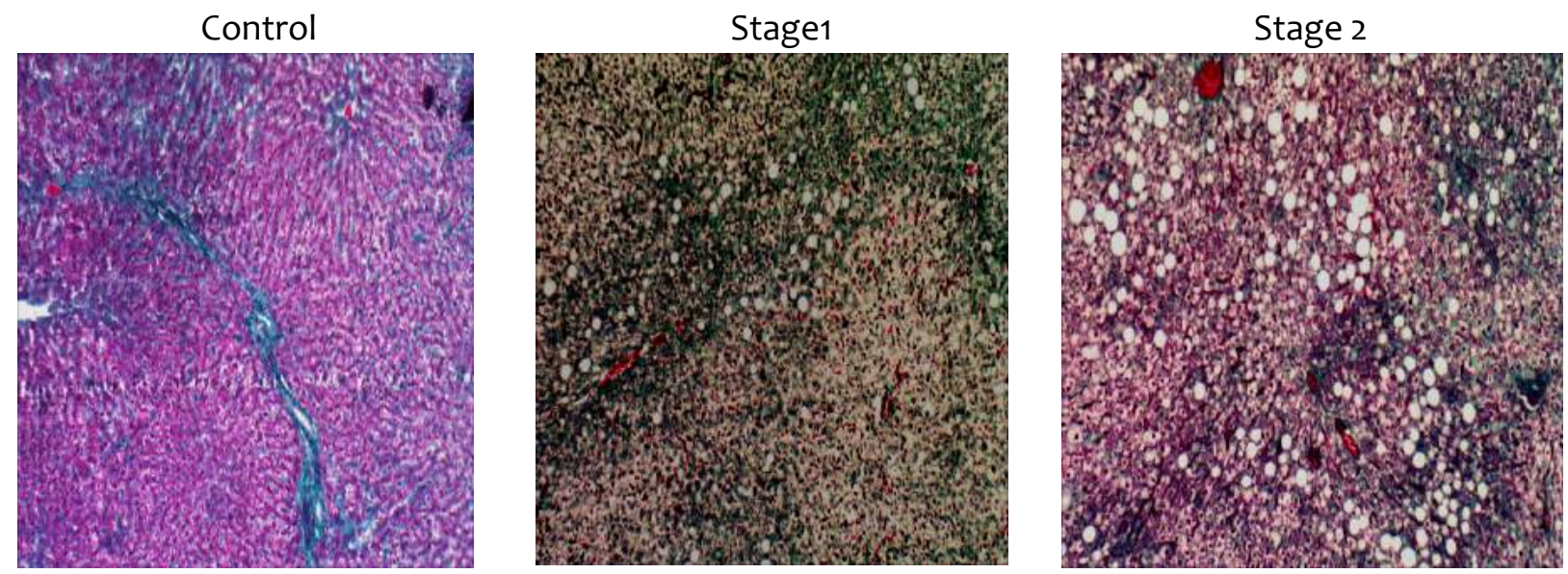

Figure 1: A) Photomicrographs showing the histopathological changes in the liver of rats from the different experimental groups. Grade 1 steatohepatitis was evident in continuous HFD 8 and 12 weeks. Grade 2 steatohepatitis at continuous HFD 16 weeks. Grade 3 steatosis, grade 2 hepatitis at cycling 16 weeks (H\&E $\mathrm{x} 100$ ).Arrows pointed to inflammatory foci B) Masson's stain Photomicrographs showing mild to moderate stages of fibrosis present in the study groups. 
A)
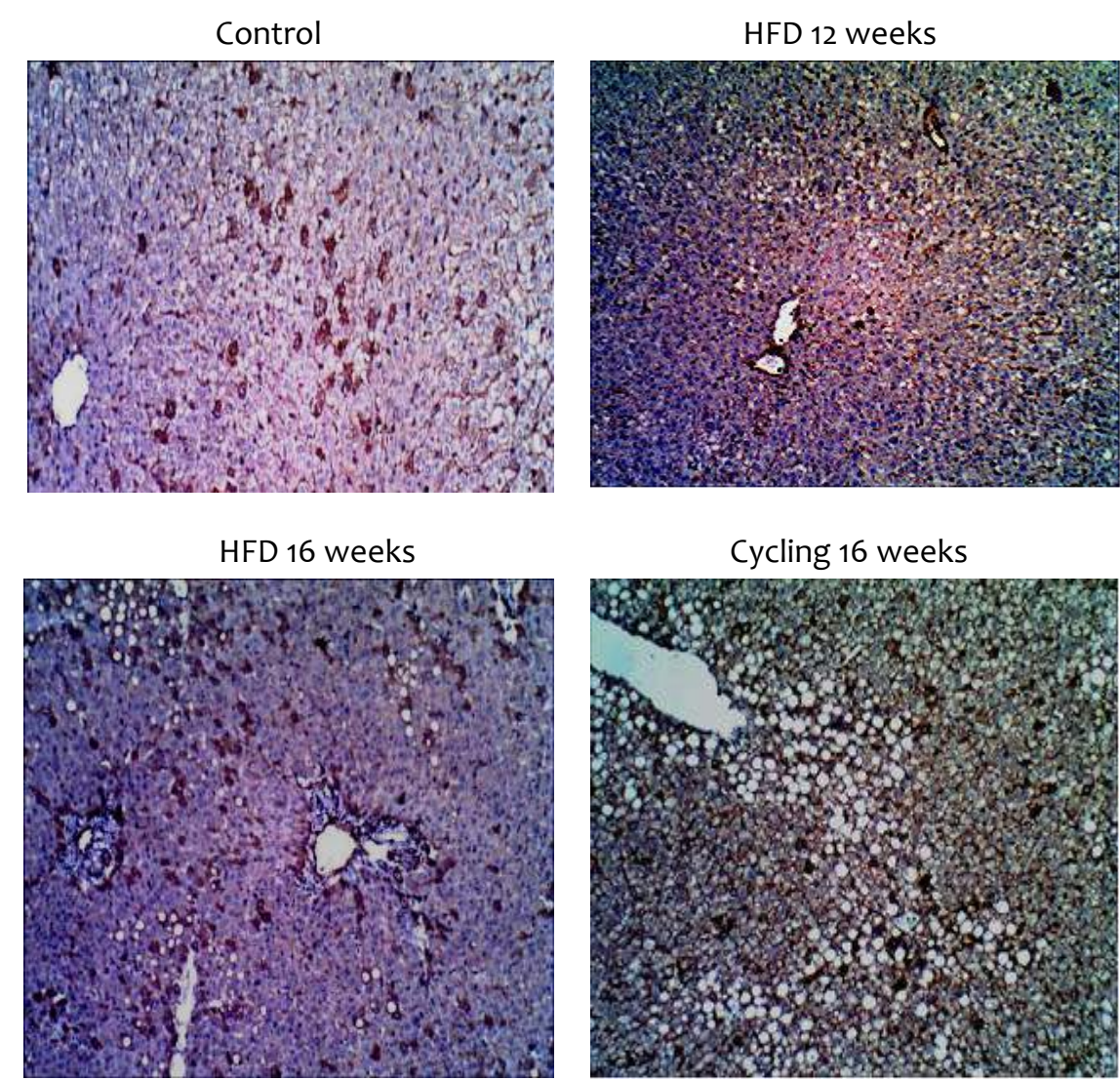

B)

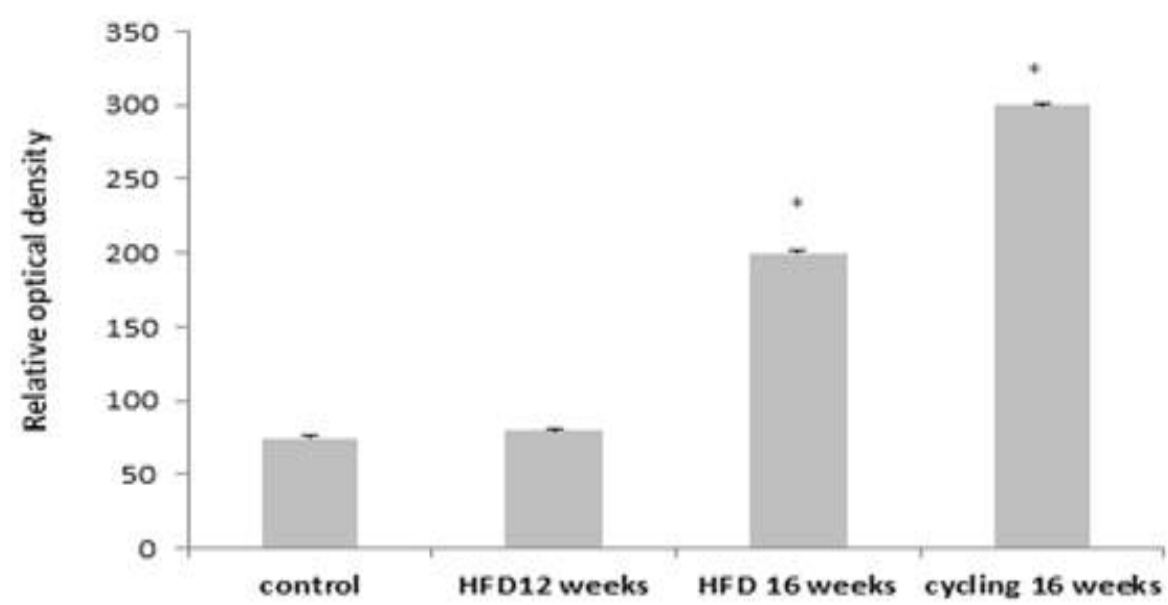

Figure 2: A) Photomicrographs showing the immunostaining for a-SMA in rat liver from significant experimental groups. There is increase in the expression of a-SMA marker in continuous HFD 16 weeks as well as cycling 16 weeks (IHC, 100X). B) Optical density for a-SMA immune-positive cells in continuous HFD 16 weeks as well as cycling 16 weeks groups relative to the control group was quantified by Image-Pro Plus 0.6 software. Values are expressed as mean \pm SEM and were analyzed using one-way ANOVA and Bonferroni posthoc test. ${ }^{*}=$ Cycling and HFD group vs. control group $8,12,16$ weeks at $P<0.05 . \dagger=C y c l i n g$ group vs. HFD 8,12,16 weeks group at $P<0.05$. \$ $\$=$ Cycling group 16 weeks vs. cycling group 8 weeks at $P<0.05$. 
This finding may be explained by lipotoxicity which is attributed to increased hepatic fat load in the setting of reduced antioxidant ${ }^{(13,34-35)}$. Such increased load, especially after diet cycling was confirmed in our study by the histopathological findings as grade 2 steatohepatitis was evident in HFD group at the end of 16 weeks, while diet-cycling group at 16 weeks revealed a more advanced grade of steatosis (grade 3) with grade 2 hepatitis. With HFD, significant elevations in total cholesterol and triglycerides in the sera of rats were observed in the current study. In agreement with our results, a significant elevation in serum triglycerides $^{(28)}$ or both serum total cholesterol and triglycerides were observed after HFD feeding ${ }^{(29-30)}$.These elevated levels were reversed after shifting to normal diet in the diet cycling group. Continuous HFD for 16 weeks shared the same lipid profile of the diet cycling group at 16 weeks. Consistently, researchers noted no long term adverse effects of cycling on lipid profile ${ }^{(36-}$ 37). Our findings can be explained under the umbrella of increased BMI and hence adiposity with increased adipose tissue releases of FFA resulting in hypertriglyceridemia $^{(38)}$ as well as hypercholesterole$\mathrm{mia}^{(39)}$. The marked increase in FFA in the diet cycling group may be due to the increased adipose tissue which is manifested by increased BMI even more than the continuous HFD group. In addition, adipose tissue is a metabolically active tissue which is responsible for production of proinflammatory cytokines ${ }^{(38)}$, most notably TNF- $\alpha$ which in turn increases FFA release ${ }^{(40)}$. In the current study, serum level of TNF- $a$, IL- 6 were significantly increased after HFD feeding at the end of all time points and these findings came on line with previous results ${ }^{(26)}$. Surprisingly, only IL-6 significantly decreased in diet cycling at12 weeks, so shifting to NCD did not decrease TNF-a high level that remained significantly higher in diet cycling group all over the study period, moreover, TNF-a level returned to overshoot again at the end of diet cycling at 16 weeks. This indicates that alternating diet caused marked elevation in TNF- $\alpha$ level even more than the elevation that was caused by continuous HFD for 16 weeks. The elevated levels of inflammatory cytokines at 12 weeks in diet cycling group despite the decrease in BMI may be due to reduction in the fat-free mass not the adipose tissue content. In the current study, a significant increase in insulin resistance after HFD was observed. This finding could be due to the up-regulation of TNF- $\alpha$ with the excessive release of FFA which may induce and potentiate IR. FFA impairs insulin mediated glucose signal at skeletal muscles leading to more deposition in the liver and more IR ensues ${ }^{(41)}$. On the other hand, high insulin levels inhibit FFA oxidation and increase the levels of toxic free FFA which causing more liver cell injury ${ }^{(42-44)}$. In the current study serum FFA concentrations were significantly higher in diet cycling group than HFD group and this change may participate in increasing the severity of liver injury in diet cycling group. Moreover, TNF$\alpha$ is an important mediator of insulin resistance due to its ability to influence the tyrosine kinase activity of the insulin receptor ${ }^{(45)}$. Also TNF-a is known to inhibit the propagation of insulin receptor initiated signals in hepatocytes. Consistently, an improvement of hepatic steatosis, inflammation and liver cell ballooning was produced by insulin ${ }^{(29,46-47)}$. Interestingly, insulin resistance causes loss of protection against TNF- $\alpha$ and reactive oxygen species ${ }^{(5,13)}$. Taken together, TNF- $\alpha$ modulation of insulin resistance in diet cycling could be a potential mechanism and a key point in our study. The currently observed elevation in oxidative stress that was detected in HFD and diet cycling groups at the end of all time points is consistent with previous re- 
ports ${ }^{(26,29)}$. The comparable elevations in hepatic lipid peroxide in HFD and diet cycling groups may be responsible for occurrence of the same stage of liver fibrosis in both groups. As the aldehyde products of Lipid peroxidation can activate hepatic stellate cells which increment collagen tissue in the liver and resulted in fibrosis. Our data also showed that diet cycling (16 weeks) induced a significant decrease in hepatic glutathione peroxidase and shifting to NCD did not ameliorate the oxidative stress parameters, moreover, their level mimics those of continuous HFD at any time point. Higher TNF-a level in diet cycling may be the key regulator of oxidative stress. It has been reported that Pentoxifylline (TNF-a inhibitor), significantly increased hepatic glutathione peroxidase content ${ }^{(48-49)}$. Collectively, these findings can suggest that, the main liver insult in diet cycling is associated with the regulation of TNF- $\alpha$. Despite of the variable grades of steatohepatitis, only mild to moderate stages of fibrosis were present in the study groups, this may be due to the relatively small duration of HFD exposure in the current study. At least continuous 12 weeks of HFD were mandatory to start fibrosis and late stages occurred after 24-48 weeks ${ }^{(13)}$. Finally, our results support the notion that diet cycling has a more deteriorating effect on the course of NAFLD than a continuous HFD. Biochemical involvement is more obvious than disturbed histological architecture.

\section{Conclusions}

The current study improved our knowledge of the effect of diet cycling on the pathogenesis of NAFLD and highlighted that HFD-induced NAFLD in rats is reproducible model, bearing almost all biochemical, histopathological aspects of human NAFLD. Diet cycling poses the highest levels of TNF- $\alpha$ as a pro-inflammatory cytokine and the lowest hepatic levels of antioxi- dant. Thus, insulin resistance, oxidative stress and steatohepatitis seem to be more evident in diet cycling group. A more detailed study to determine the exact body composition, the exact site of IR and the long-term consequences of diet alterations on liver is mandatory.

\section{Conflict of interest}

The authors declare that they have no conflict of interest.

\section{References}

1. Suzuki A, Angulo P, Lymp J, St Sauver J, Muto A, Okada T, Lindor K. Chronological development of elevated aminotransferases in a nonalcoholic population. Hepatology 2005; 41 (1): 64-71.

2. Hamaguchi $M$, Kojima T, Takeda N, Nakagawa $\mathrm{T}$, Taniguchi $\mathrm{H}$, Fujii $\mathrm{K}$, Omatsu $\mathrm{T}$, Nakajima T, Sarui H,Shimazaki M, Kato T, Okuda J, Ida K. The metabolic syndrome as a predictor of non-alcoholic fatty liver disease. Ann Intern Med 2005; 143 (10) : 722-728.

3. Whalley S, Puvanachandra $P$, Desai $A$, Kennedy $\mathrm{H}$. Hepatology outpatient service provision in secondary care: a study of liver disease incidence and resource costs. Clin Med 2007; 7 (2): 119-124.

4. Lee JY, Kim KM, Lee SG, Yu E, Lim YS, Lee HC, Chung YH, Lee YS, Suh DJ. Prevalence and risk factors of non-alcoholic fatty liver disease in potential living liver donors in Korea: a review of 589 consecutive liver biopsies in a single center. $J$ Hepatol 2007; 47 (2): 239- 244.

5. Bellantani S, Saccoccio G, Masutti F, Crocè LS, Brandi G, Sasso F, Cristanini G, Tiribelli $C$. Prevalence and risk factors for hepatic steatosis in northern Italy. Ann Intern Med 2000; 132 (2):112- 117.

6. Day CP, James OF. Steatohepatitis: a tale of two "hits". Gastroenterology 1998; 114 (4): 842-845.

7. Duvnjak M, Lerotic I, Barsic N, Tomasić $V$, Virović Jukić L, Velagić V. Pathogenesis and management issues for nonalcoholic 
fatty liver disease. World J. Gastroenterol 2007; 13 (34): 4539-4550.

8. Solga $S$, Alkhuraishe AR, Clark JM, Torbenson M, Greenwald A, Diehl AM, Magnuson T. Dietary composition and nonalcoholic fatty liver disease. Dig Dis Sci. 2004; 49(10): 1578-1583.

9. Zelber-Sagi S1, Nitzan-Kaluski D, Goldsmith R, Webb M, Blendis L, Halpern Z, Oren $\mathrm{R}$. Long term nutritional intake and the risk for non-alcoholic fatty liver disease (NAFLD): A population based study. J. Hepatol 2007; 47(5): 711-717.

10. Motomura W, Yoshizaki T, Takahashi N, Kumei S, Mizukami Y, Jang SJ, Kohgo Y. Analysis of vanin1 upregulation and lipid accumulation in hepatocytes in response to a high fat diet and free fatty acids. J. Clin. Biochem. Nutr 2012; 51(3): 163-169.

11. Elizabeth LY, Jeffrey BS, Joel EL. Nonalcoholic fatty liver disease: epidemiology, pathophysiology, diagnosis and treatment. Paediatr. Child Health 2010; 20: 26-29.

12. Duarte FO, Sene-Fiorese M, Manzoni MS, de Freitas LF, Cheik NC, Garcia de Oliveira Duarte AC,Nonaka KO, Dâmaso A. Caloric restriction and refeeding promoted different metabolic effects in fat depots and impaired dyslipidemic profile in rats. Nutrition 2008; 24 (2): 177-186.

13. Xu ZJ, Fan JG, Ding XD, Qiao L, Wang GL. Characterization of High-Fat, DietInduced, Non-alcoholic Steatohepatitis with Fibrosis in Rats. Dig Dis Sci 2010; 55 (4): 931-940.

14. Novelli EL, Diniz YS, Galhardi CM, Ebaid GM, Rodrigues HG, Mani F, Fernandes AA, Cicogna AC,Novelli Filho JL. Anthropometrical parameters and markers of obesity in rats. Lab Anim 2007; 41 (1): 111119.

15. Matthews DR, Hosker JP, Rudenski AS, Naylor BA, Treacher DF, Turner RC. Homeostasis model assessment: insulin resistance and beta-cell function from fasting plasma glucose and insulin concentrations in man. Diabetologia 1985; 28 (7): 412-419.

16. Draper HH, Hadley M. Malondialdehyde determination as index of lipid peroxida- tion. Methods Enzymol 1990; 186: 421431.

17. Woolliams JA, Wiener G, Anderson PH, McMurray $\mathrm{CH}$. Variation in the activities of glutathione peroxidase and superoxide dismutase and in the concentration of copper in the blood in various breed crosses of sheep. Res Vet Sci 1983; 34 (3): 253-256.

18. Aebi H. Catalase in vitro. Methods Enzymol 1984; 105: 121-126.

19. Beutler E, Duron O, Kelly BM. Improved method for the determination of blood glutathione. J Lab Clin Med 1963; 61: 882888.

20. Dixon JB, Bhathal PS, Hughes NR, O'Brien $\mathrm{PE}$. Nonalcoholic fatty liver disease: improvement in liver histological analysis with weight loss. Hepatology 2004; 39 (6): 1647-1654.

21. Williams R. Global changes in liver disease. Hepatology 2006; 44: 521-526. doi:10.1002/hep.21347.

22. Angulo P. Gl epidemiology: nonalcoholic fatty liver disease. Aliment Pharmacol Ther. 2007; 25 (8): 883-889.

23. Fan JG, Farrell GC. Epidemiology of nonalcoholic fatty liver disease in China. J Hepatol 2009; 50 (1): 204-210.

24. Ong JP, Younossi ZM. Epidemiology and natural history of NAFLD and NASH. Clin Liver Dis 2007; 11 (1): 1-16. doi:10.1016/j.cld.2007.02.009.

25. Vilar L, Oliveira CP, Faintuch J, Mello ES, Nogueira MA, Santos TE, Alves VA, Carrilho FJ. High-fat diet: A trigger of non-alcoholic steatohepatitis? Preliminary findings in obese subjects. Nutrition 2008; 24 (11-12): 1097-1102.

26. Yalniz M, Bahcecioglu IH, Kuzu N, Celebi $\mathrm{S}$, Ataseven $\mathrm{H}$, Ustündağ $\mathrm{B}$, Ozercan $\mathrm{IH}$, Sahin K. Amelioration of steatohepatitis with pentoxifylline in a novel nonalcoholic steatohepatitis model induced by highfat diet. Dig Dis Sci 2007; 52 (9): 23802386.

27. Gao ZQ, Lu FE, Dong H, Xu LJ, Wang KF, Zhou X. Study on therapeutic effects of metformin on rat fatty livers induced by high fat feeding. Zhonghua Gan Zang Bing Za Zhi 2005; 13 (2): 101-104. 
28. Seböková E1, Kürthy $M$, Mogyorosi $T$, Nagy K, Demcáková E, Ukropec J, Koranyi L, Klimes I. Comparison of the extrapancreatic action of BRX-220 and pioglitazone in the high-fat diet-induced insulin resistance. Ann N Y Acad Sci 2002; 967; 424-430.

29. Hsiao PJ, Hsieh TJ, Kuo KK, Hung WW, Tsai KB, Yang $\mathrm{CH}$, Yu ML, Shin SJ. Pioglitazone retrieves hepatic antioxidant DNA repair in a mice model of high fat diet. BMC Mol Biol 2008; 9: 82.

30. Pan M, Song YL, Xu JM, Gan HZ. Melatonin ameliorates nonalcoholic fatty liver induced by high-fat diet in rats. J Pineal Res 2006; 41 (1): 79-84.

31. MacLean PS, Higgins JA, Johnson GC, et al.: Metabolic adjustments with the development, treatment, and recurrence of obesity in obesity-prone rats. Am J Physiol Regul Integr Comp Physiol 2004; 287: R288-297.

32. MacLean PS, Higgins JA, Jackman MR, Johnson GC, Fleming-Elder BK, Wyatt HR, Melanson EL, Hill JO. Peripheral metabolic responses to prolonged weight reduction that promote rapid, efficient regain in obesity-prone rats. Am J Physiol Regul Integr Comp Physiol 2006; 290 (6): R15771588.

33. Strohacker K, Carpenter KC, Mcfarlin BK. Consequences of Weight Cycling: An Increase in Disease Risk? Int J Exerc Sci. 2009; 2(3): 191-201.

34. London RM, George J. Pathogenesis of NASH: animal models. Clin Liver Dis 2007; 11 (1): 55-74.

35. Nanji AA. Animal models of nonalcoholic fatty liver disease and steatohepatitis. Clin Liver Dis 2004; 8 (3): 559-574.

36. Li Z, Hong K, Wong E, Maxwell M, Heber D. Weight cycling in a very low-calorie diet programme has no effect on weight loss velocity, blood pressure and serum lipid profile. Diabetes Obes Metab 2007; 9 (3): 379-385.

37. Graci S, Izzo G, Savino S, Cattani L, Lezzi G, Berselli ME, Balzola F, Liuzzi A, Petroni ML. Weight cycling and cardiovascular risk factors in obesity. Int $\mathrm{J}$ Obes Relat Metab Disord 2004; 28 (1): 65-71.
38. Farrell GC, Larter CZ. Nonalcoholic fatty liver disease: from steatosis to cirrhosis. Hepatology 2006; 43(2 suppl 1): S99S112.

39. Brinton EA, Eisenberg S, Breslow JL. Increased apo A-I and apo A-II fractional catabolic rate in patients with low high density lipoprotein-cholesterol levels with or without hypertriglyceridemia. J Clin Invest 1991; 87 (2): 536-544.

40. Palomo I, Alarcon M, Moore-Carrasco R, Argilés JM. Hemostasis alterations in metabolic syndrome (review). Int J Mol Med 2006; 18 (5): 969-974.

41. Avramoglu RK, Basciano H, Adeli K. Lipid and lipoprorein dysregulation in insulin resistant states. Clin Chim Acta 2006; 368 (1-2): 1-19.

42. Cavallo-Perin P, Cassader M, Bozzo C, Bruno A, Nuccio P, Dall'Omo AM, Marucci $M$, Pagano G. Mechanism of insulin resistance in human liver cirrhosis: Evidence of a combined receptor and postreceptor defect. J Clin Invest 1985; 75 (5): 1659-65.

43. Taylor R, Heine RJ, Collins J, James OF, Alberti KG. Insulin action in cirrhosis. Hepatology 1985; 5 (1): 64-71.

44. Berson A, De Beco V, Lettbon P, Robin MA, Moreau C, El Kahwaji J, Verthier N, Feldmann G, Fromenty B, Pessayre D. Steatohepatitis-inducing drugs cause mitochondrial dysfunction and lipid peroxidation in rat hepatocytes. Gastroenterology 1998; 114 (4): 764-774.

45. Hotamisligil GS, Peraldi P, Budavari A, Ellis R, White MF, Spiegelman BM. IRS-1mediated inhibition of insulin receptor tyrosine kinase activity in TNF-alpha and obesity-induced insulin resistance. Science 1996; 271 (5249): 665-668.

46. Fujita K, Yoneda M, Wada K, Mawatari H, Takahashi H, Kirikoshi H, Inamori $M$, Nozaki Y, Maeyama S,Saito S, Iwasaki T, Terauchi Y, Nakajima A. Telmisartan, an angiotensin II type 1 receptor blocker, controls progress of nonalcoholic steatohepatitis in rats. Dig Dis Sci 2007; 52 (12): 3455-3464.

47. Xu P, Zhang XG, Li YM, Yu CH, Xu L, Xu $G Y$. Research on the protection effect of 
pioglitazone for non-alcoholic fatty liver disease (NAFLD) in rats. J Zhejiang Univ Sci 2006; 7 (8): 627-633.

48. Koppe SW, Sahai A, Malladi P, Whitington PF, Green RM. Pentoxifylline attenuates steatohepatitis induced by the methionine choline deficient diet. J
Hepatol 2004; 41 (4): 592-598.

49. Zaitone S, Hassan N, El-Orabi N, El-Awady el-S. Pentoxifylline and melatonin in combination with pioglitazone ameliorate experimental non-alcoholic fatty liver disease. Eur J Pharmacol. 2011; 662(13):70-77. 